\title{
Dysbiosis of Oral Microbiota Associated with Palmoplantar Pustulosis
}

\author{
Yasunari Kageyama, ${ }^{a}$ Yutaka Shimokawa ${ }^{a}$ Kimihiko Kawauchi ${ }^{a}$ \\ Masafumi Morimoto ${ }^{a}$ Koichi Aida ${ }^{a}$ Tetsu Akiyama ${ }^{c}$ Tsutomu Nakamurac \\ a Takanawa Clinic, Minato-ku, Tokyo, Japan; ${ }^{\text {bTokai University Hospital, Isehara-shi, Kanagawa, Japan; ' } L a b o r a t o r y ~ o f ~}$ \\ Molecular and Genetic Information, Institute for Quantitative Biosciences, The University of Tokyo, Tokyo, Japan
}

\section{Keywords}

Palmoplantar pustulosis - Oral microbiome - Dysbiosis .

Periodontal diseases

\begin{abstract}
Background: Dysbiosis of oral microbiota is implicated not only in oral inflammatory lesions, but also in a variety of extraoral diseases. The etiology of palmoplantar pustulosis (PPP) remains unclear; however, it has been suggested that chronic inflammation caused by periodontopathic bacterial infection may play a role. Objectives/Methods: To determine whether patients with PPP have altered diversity and composition of oral microbiota, we conducted the 16S rDNA analysis using saliva samples collected from 21 outpatients with PPP and 10 healthy individuals. Results: We found that the proportion of bacteria in the phylum Proteobacteria was significantly lower in PPP patients $(p=0.025)$. At the genus level, patients with PPP had a significantly lower abundance of Neisseria ( $p=0.014$ ), which best accounted for the observed decrease in Proteobacteria. We also identified multiple minor genera and species that were represented at a significantly higher level in the PPP group, several of which have been associated with periodontal diseases. Conclusion: Our results suggest a possible link between PPP and dysbiosis of oral microbiota, particularly the lower abun-
\end{abstract}

karger@karger.com www.karger.com/drm

Karger $\stackrel{\text { ' }}{5}$

GOPEN ACCESS
(C) 2020 The Author(s)

Published by S. Karger AG, Basel

This is an Open Access article licensed under the Creative Commons Attribution-NonCommercial-4.0 International License (CC BY-NC) (http://www.karger.com/Services/OpenAccessLicense), applicable to the online version of the article only. Usage and distribution for commercial purposes requires written permission. dance of Neisseria, the most predominant genus of Proteobacteria in healthy oral microbiota. Probiotics that improves oral dysbiosis may be beneficial for patients with PPP as an adjunctive therapy.

(c) 2020 The Author(s)

Published by S. Karger AG, Basel

\section{Introduction}

The human oral cavity harbors more than 700 different species of resident microorganisms and is the second most diverse microbial community in the body. The growth of periodontal pathobionts, local odontogenic infection, and chronic inflammation have been linked to changes in the diversity and composition (dysbiosis) of the oral microbiota $[1,2]$. In addition, oral dysbiosis may also contribute to a variety of systemic or autoimmune diseases [3-6], including atherosclerosis [7, 8], coronary artery disease $[9,10]$, diabetes mellitus $[11,12]$, rheumatoid arthritis $[13,14]$, systemic lupus erythematosus [15, $16]$, and Sjögren's syndrome $[17,18]$. The oral microbiome serves as a source of pathogenic microorganisms, bioactive metabolites, and proinflammatory molecules for extraoral tissues. 
Palmoplantar pustulosis (PPP) is a refractory skin disorder in which crops of multiple sterile pustules appear recurrently and bilaterally on the palms and soles. The pustules form erythematous, scaly plaques with chronic pain and/or itch, which seriously impairs the quality of life of the patients. The etiological factors for PPP remain unclear; however, it has been suggested that smoking and dental metal hypersensitivity trigger and/or exacerbate PPP [19-22]. Furthermore, accumulating evidence suggests that chronic odontogenic infection, in particular periapical lesions, has a close pathogenetic relationship with PPP [23-25].

To evaluate the role of oral microbiota in the pathogenesis of PPP, we conducted a comparative analysis of oral microbial profiles in patients with PPP and healthy individuals. The results of this study highlight specific oral dysbiosis in patients with PPP and suggest the possibility of adjunctive oral probiotics that improves the taxonomic composition of the oral microbial community in combination with the current pharmacotherapy for PPP.

\section{Materials and Methods}

For further details, see the online supplementary material (see www.karger.com/doi/10.1159/000511622 for all online suppl. material, ) (Fig. 1). The main characteristics of PPP patients are summarized in online supplementary Table 1.

\section{Results}

Periodontal Health Status of the Subjects

Periodontal disease (probing depth $\geq 4 \mathrm{~mm}$ ) was found in $42.1 \%$ of the PPP patients and half of the healthy controls (HC), and the difference was not significant ( $p=$ 0.714 in two-tailed Fisher's exact test). No significant difference in the number (5.75 [PPP] vs. $2.00[\mathrm{HC}], p=$ 0.0789 in two-tailed Welch's $t$ test) and the mean depth (4.41 mm [PPP] vs. $4.00 \mathrm{~mm}[\mathrm{HC}], p=0.0931$ in twotailed Welch's $t$ test) of periodontal pockets between PPP and $\mathrm{HC}$ was found. We thus reasoned that the enrolled PPP and HC subjects had a similar periodontal health status.

\section{PPP Patients Have Dysbiosis of Oral Microbiota}

In next-generation sequencing-based $16 \mathrm{~S}$ rDNA amplicon analysis, averages (SD) of 17,621 (4,683; PPP) and 20,805 (4,985; HC) clean reads were obtained per sample,

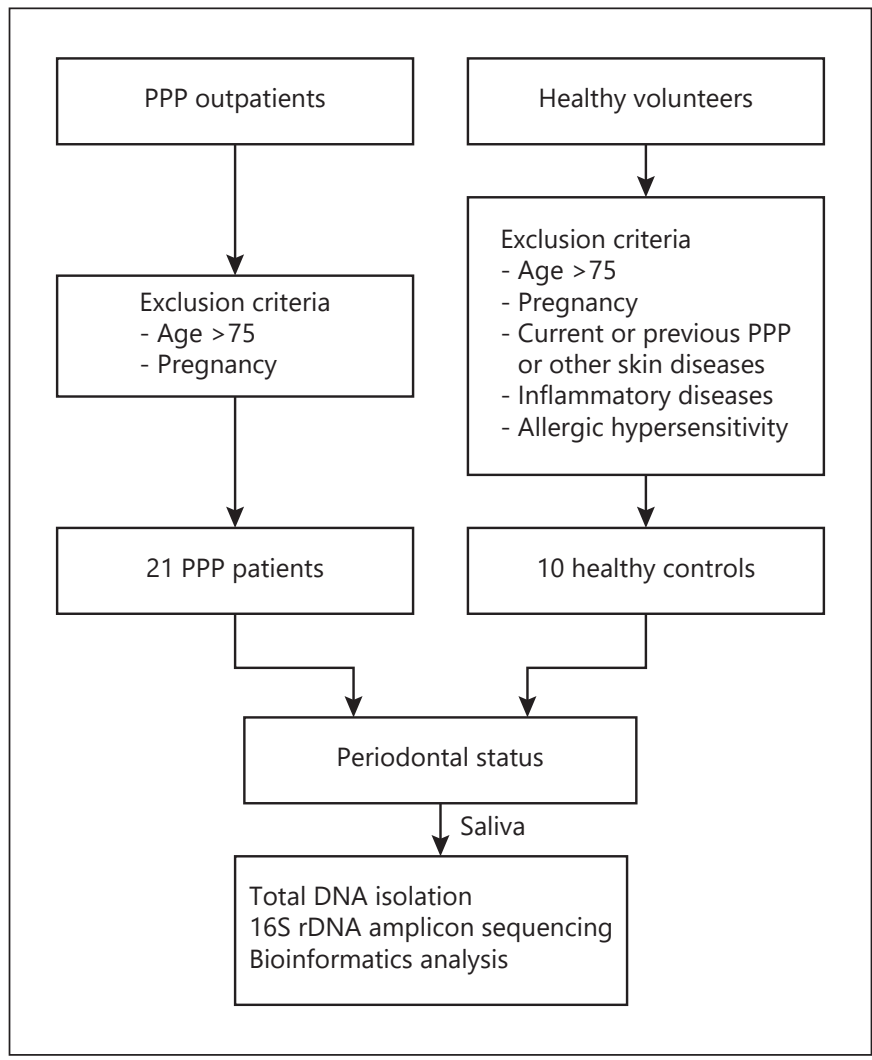

Fig. 1. Flowchart of the Materials and Methods.

clustered into operational taxonomic units with $97 \%$ similarity, and assigned taxonomically. Of 198 operational taxonomic units (OTUs) obtained, 103 OTUs (52.0\%) were assigned taxonomically at the genus level and 42 OTUs (21.2\%) at the species level. A total of 14 phyla, 22 classes, 36 orders, 59 families, 96 genera, and 42 species were represented in the saliva samples (online suppl. Table 2). Generally, biodiversity of a microbial community has been assessed from 2 aspects: species diversity within a single ecosystem ( $\alpha$-diversity) and differences in overall diversity between different environments ( $\beta$-diversity). There was no significant difference in the standard estimates of $\alpha$-diversity between PPP patients and HC, as measured by species richness $(p=0.521$ in two-tailed Welch's $t$ test; Fig. 2a), evenness ( $p=0.601$ in two-tailed Welch's $t$ test; Fig. $2 \mathrm{~b}$ ), and diversity ( $p=0.998$ in twotailed Welch's $t$-test; Fig. $2 c ; p=0.381$ in two-tailed Welch's $t$ test; Fig. 2 d). However, $\beta$-diversity analysis revealed a clear separation between the PPP and HC microbial communities at the phylum and the genus levels (Fig. 2e, f), suggesting that PPP patients and $\mathrm{HC}$ have distinct oral microbial compositions. 


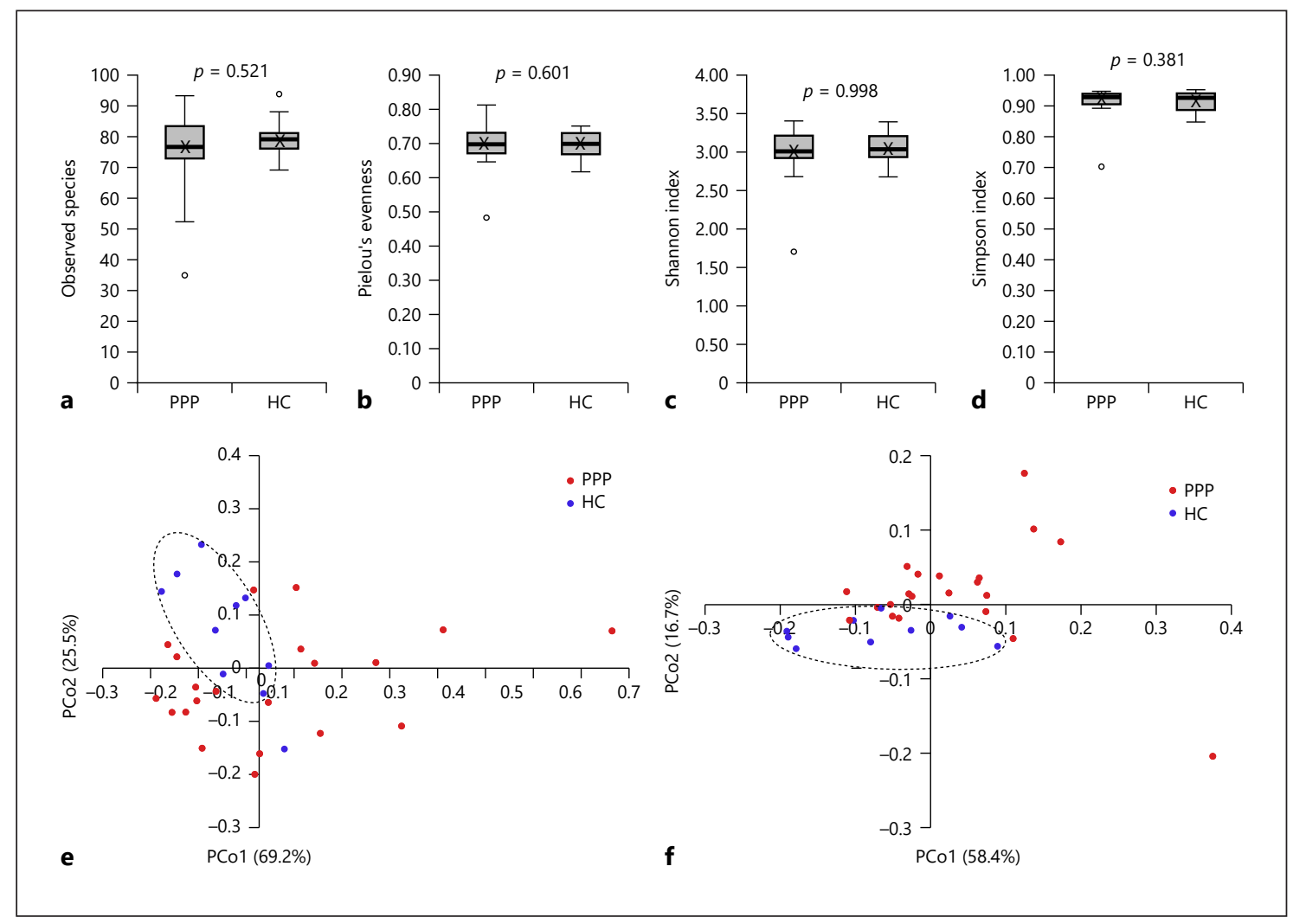

Fig. 2. $\alpha$ - and $\beta$-Diversity comparisons of the oral microbiomes of the PPP and HC groups. a-d $\alpha$-Diversity comparisons based on the species richness (a), species evenness (b), and species diversity (c, d). The mean values and outliers are shown by cross marks and open circles, respectively. Species richness, defined as the number of species identified in each sample, is the simplest measure of biodiversity. Pielou's evenness is a good measure of "relative evenness" for each community. Species diversity (Shannon index and
Simpson index) is a more complex measure that takes into account both species richness and evenness. e, $\mathbf{f} \beta$-Diversity comparisons using principal coordinate analysis at the phylum (e) and the genus (f) levels. The proportion of variance explained by each principal coordinate (PCo) axis is denoted in the corresponding axis label. PPP and HC groups show clear separation as shown by dashed circles.

\section{Decreased Proportion of Proteobacteria in PPP}

We next investigated the taxonomic composition of the oral microbiota of PPP patients and HC. At the phylum level, a significant decrease in the proportion of Proteobacteria ( $p=0.0251$ in two-tailed Welch's $t$ test) and a significant increase in the proportion of Synergistetes ( $p=0.0485$ in two-tailed Welch's $t$ test) were observed in patients with PPP (Fig. 3a-c). Proteobacteria, along with Bacteroidetes and Firmicutes, was the most predominant phylum in HC; however, in patients with PPP, the relative abundance of Proteobacteria was decreased to nearly half that of Bacteroidetes and Firmicutes (Fig. 3a). Hierarchical cluster analysis revealed that PPP and HC were separated into 4 different clusters based on the phylum composition of the oral microbiota: one $\mathrm{HC}$-specific cluster characterized by a higher proportion of Proteobacteria and 3 PPP clusters with higher relative abundance of Bacteroidetes or Firmicutes (Fig. 3d).

\section{Reduced Abundance of Neisseria in PPP}

The differences in oral microbiota composition were further confirmed at the genus level. We found that patients with PPP had a significantly reduced abundance of the genus Neisseria than HC did ( $p=0.0140$ in two-tailed Welch's $t$ test; Fig. 4a, b). Neisseria was the most predominant genus within Proteobacteria in healthy oral microbiota (Fig. 4a), and this reduction accounted for a large percentage of the observed decrease in Proteobacteria in PPP patients (Fig. 4b). Furthermore, the relative abundance of the rare genera within Firmicutes and Synergistetes was significantly increased in PPP patients: $D i$ alister ( $p=0.0356$ in two-tailed Welch's $t$ test), Schwartz- 


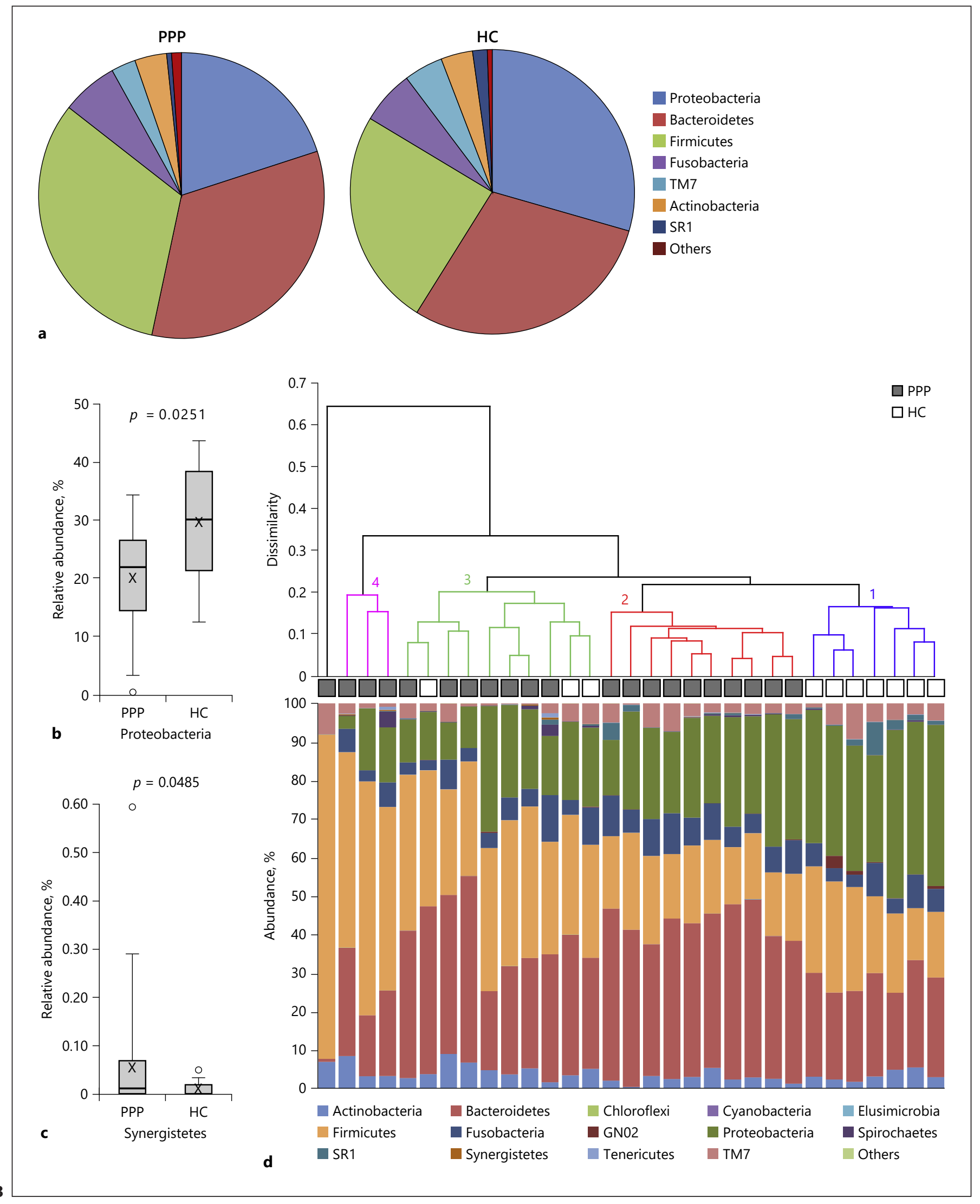

(For legend see next page.) 


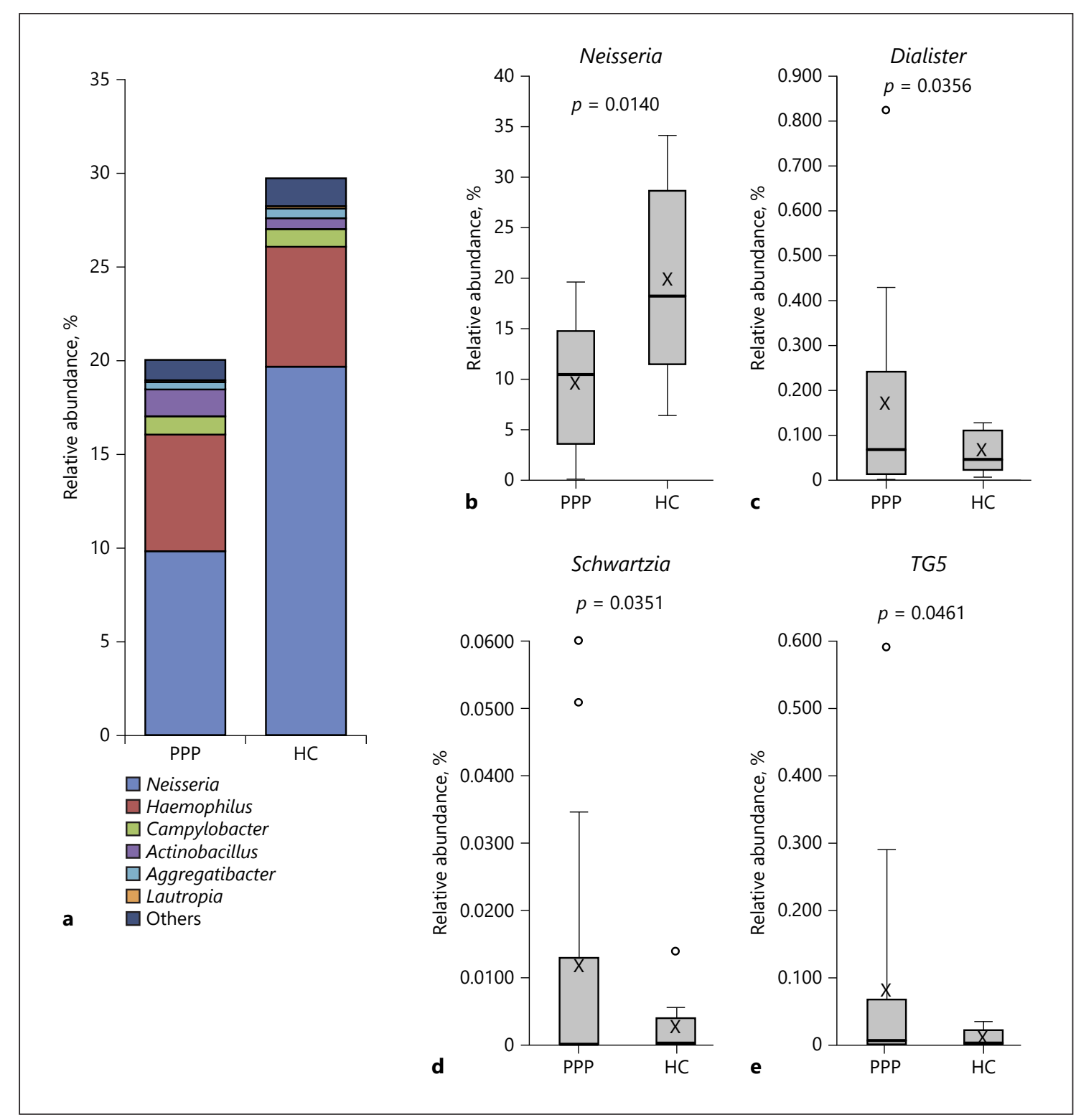

Fig. 4. Taxonomic composition analysis of the oral microbiomes of the PPP and HC groups at the genus level. a Comparisons of the genus-level abundance within Proteobacteria of PPP and $\mathrm{HC}$ are shown in bar plots. The genera representing $<0.1 \%$ of the total oral microbes of HC are included in "others." b-e Comparisons of the

Fig. 3. Taxonomic composition analysis of oral microbiomes of the PPP and HC groups at the phylum level. a The relative abundances of oral bacterial phyla of PPP and HC are represented by pie charts. The phyla representing $<0.5 \%$ of the total oral microbes of $\mathrm{HC}$ are included in "others." b, c Box-and-whisker plots show the comparisons of the relative abundance of the phyla with significant differences between PPP and HC. The mean values and outliers are shown by cross marks and open circles, respectively. d Hierarchical clustering of the phylum-level composition of the oral bacterial community of PPP and HC. The clustering results are repre- relative abundance of the genera with significant differences between PPP and HC. The genera within Proteobacteria (b), Firmicutes $(\mathbf{c}, \mathbf{d})$, and Synergistetes (e) are shown in box-and-whisker plots. The mean values and outliers are shown by cross marks and open circles, respectively.

sented as a dendrogram. Bar plots indicate the phylum-level composition of oral microbiota of each subject. Four different clusters (1-4 in the dendrogram) were identified. The cluster highlighted in blue corresponds to the $\mathrm{HC}$ microbiome characterized by a higher relative abundance of Proteobacteria. The red cluster represents a group of PPP patients harboring a higher relative abundance of Bacteroidetes. The clusters shown in green and magenta include PPP patients with a higher relative abundance of Firmicutes. 


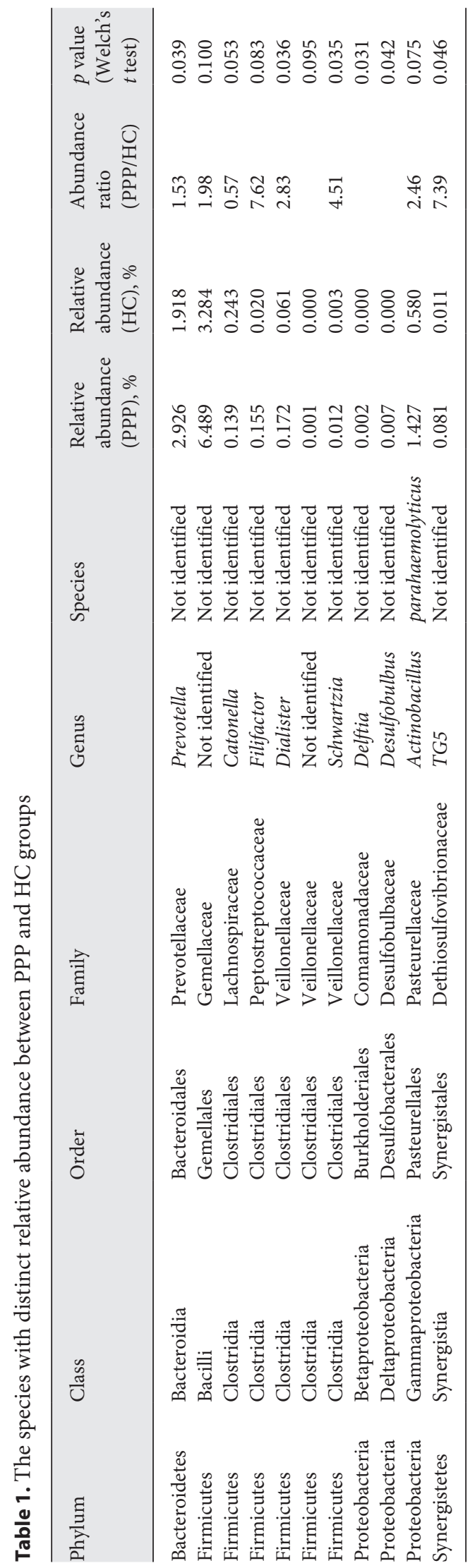

ia ( $p=0.0351$ in two-tailed Welch's $t$ test) and TG5 ( $p=$ 0.0461 in two-tailed Welch's $t$ test), all of which have been associated with periodontal disease and endodontic lesions (Fig. 4c-e) [26-28].

\section{Higher Proportion of Periodontopathic Bacterial \\ Species in PPP Patients}

We found 11 species with distinct relative abundance between PPP patients and HC (Table 1). Several of these species, such as Prevotella sp., Dialister sp., Schwartzia sp., and TG5 sp., were members of genera that are closely associated with odontogenic infection [26-29]. Three species were detected only in patients with PPP, 2 of which (unidentified species within the family Veillonellaceae and Desulfobulbus sp.) belong to taxa known as periodontal pathobionts $[28,30]$. The overall results are illustrated schematically in Figure 5.

\section{Post Hoc Power Analysis}

Twenty-one PPP patients and $10 \mathrm{HC}$ were enrolled in this study. In the analysis of Proteobacteria, the mean proportion and the SD were 20.0 and 9.56 (PPP) versus 29.7 and $9.92(\mathrm{HC})$, respectively. The effect size $(d)$ was calculated to be 1.00. Similarly, in the analysis of Neisseria, the mean proportion and the SD were 9.82 and 6.23 (PPP) versus 19.7 and $9.50(\mathrm{HC})$, respectively. The effect size $(d)$ was calculated to be 1.33 . On the basis of these statistics, we obtained the statistical power values of 0.711 (Proteobacteria) and 0.917 (Neisseria).

\section{Discussion/Conclusion}

A significantly lower proportion of Proteobacteria was observed in PPP patients and appears largely attributable to the reduction of the genus Neisseria, although no specific species responsible for the decrease in Neisseria was identified in this study. Notably, an abundance of Neisseria is related to healthy periodontal conditions [31-34]. Most species within Neisseria, except for patho-

Fig. 5. Schematic summarizing the shift in oral bacteria in PPP patients. Taxa in each taxonomic hierarchy are shown by circles. The average relative abundance of the taxon in PPP (the numerical value in or below the circle) is represented by the size of the circle. Changes in the relative abundance of the taxa between PPP and $\mathrm{HCC}$ are expressed as the base 2 logarithm of the ratio of the average relative abundance ( $\mathrm{PPP} / \mathrm{HC}$ ). The weight of the outline for each circle represents the statistical significance of the taxon (twotailed Welch's $t$ test) between PPP and HC.

(For figure see next page.) 


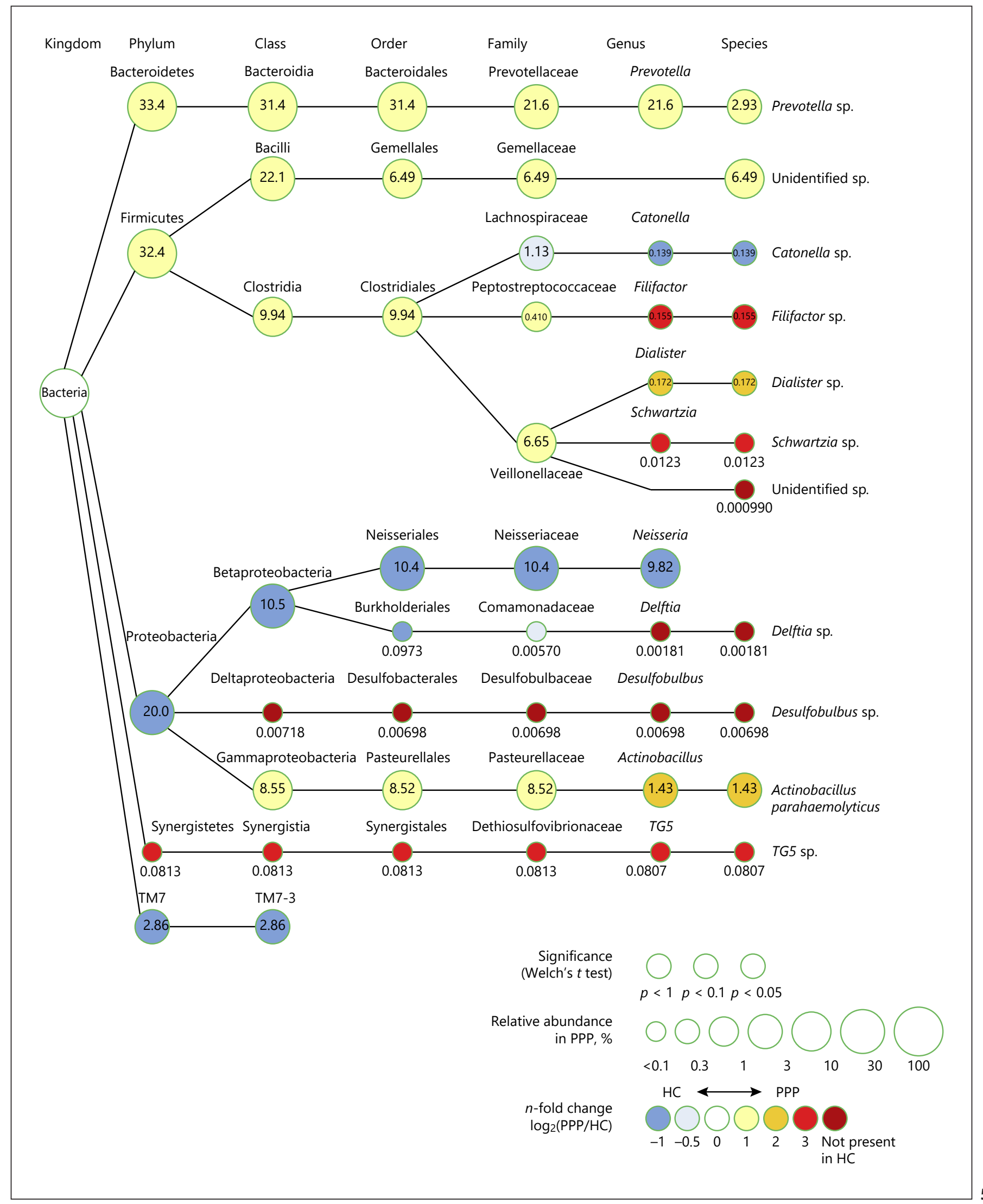

Oral Dysbiosis in Palmoplantar Pustulosis 
genic $N$. gonorrhoeae and N. meningitides, are nonpathogenic commensal bacteria in the human oral cavity. We speculate that the reduction of resident Neisseria permits the colonization and/or growth of other bacteria directly involved in PPP pathogenesis; these may include the periodontal pathogenic taxa that were found to be increased in PPP patients in this study. Neisseria is a dominant producer of acetaldehyde in the oral cavity $[35,36]$. Alternatively, Neisseria is an important contributor to the oral bacteria-mediated reduction of nitrate $\left(\mathrm{NO}_{3}{ }^{-}\right)$to nitrite $\left(\mathrm{NO}_{2}^{-}\right)[37,38]$, which can be converted to physiologically active nitric oxide (NO) in the stomach. Dysregulation of this $\mathrm{NO}_{3}{ }^{-}-\mathrm{NO}_{2}{ }^{-}-\mathrm{NO}$ pathway may cause PPP.

The phylum-level analysis also suggested that PPP was associated with a significantly higher proportion of Synergistetes, implicated in periodontitis and peri-implantitis [39-41]. The genus TG5, which was found to be significantly increased in PPP patients, has a close phylogenetic relationship with cluster A species, a periodontopathic bacterial group within the phylum Synergistetes [42-44]. Synergistetes has a potential role in oral dysbiosis through the generation of cyclodipeptide metabolites with quorum-sensing and/or bactericidal/bacteriostatic activity [45]. This induction of dysbiosis by Synergistetes may also be associated with PPP.

Although we could not find the causality between oral dysbiosis and PPP in this study, our findings suggest the possibility of oral probiotics that supplements Neisseria and/or improves oral dysbiosis as an add-on therapy. In fact, previous randomized controlled trials have demonstrated that oral probiotics using lozenges containing probiotic bacterial strains has beneficial effects on oral microbiome [46-48].

The small numbers of patients and controls examined in this study may limit its conclusions and generalizability, although the sample size yielded statistical power values at the recommended levels in a post hoc power analysis. In addition, we could find no evidence to suggest the causal relationship between oral dysbiosis and PPP in this study. Further confirmation studies with larger cohorts are needed to establish the causality. Mechanistic investigation will also be essential for a better understanding of the impact of periodontal disease and oral microbiome on PPP. Oral dysbiosis can affect the gut microbiota directly through saliva and indirectly through blood flow [49]; however, there have been no reports on the intestinal microbiome of PPP patients. Further comparative studies are required to elucidate whether PPP patients have specific dysbiosis of gut microbiota.
In conclusion, we found dysbiosis of oral microbiota, particularly, the significant decrease in the genus Neisseria and the concomitant increase in bacteria within the periodontopathic taxa, in the PPP cohort.

\section{Key Message}

Microbiome analysis reveals altered composition of oral microbiota in patients with palmoplantar pustulosis.

\section{Statement of Ethics}

This case-control observational study was carried out in accordance with the Code of Ethics of the World Medical Association (Declaration of Helsinki) and the strengthening of the reporting of observational studies in epidemiology (STROBE) guidelines. Ethical approval for this study was obtained from the Institutional Review Board of Takanawa Clinic (approval No.: 2016-1). A signed informed consent form was obtained from each participant prior to inclusion in this study.

\section{Conflict of Interest Statement}

Y.K., Y.S., K.K., M.M., and K.A. are employees of Takanawa Clinic (Tokyo, Japan). T.A. and T.N. have advisory roles in conducting clinical research in Takanawa Clinic.

\section{Funding Sources}

This research did not receive any specific grant from funding agencies in the public, commercial, or not-for-profit sectors.

\section{Author Contributions}

Y.K., Y.S., K.K., M.M., and K.A. contributed to conception and design, contributed to data analysis and interpretation, and critically revised the manuscript. T.A. contributed to conception and design and critically revised the manuscript. T.N. contributed to conception and design, contributed to data analysis and interpretation, and drafted the manuscript. All authors have read and approved the final paper. 


\section{References}

1 Kinane DF, Stathopoulou PG, Papapanou PN. Periodontal diseases. Nat Rev Dis Primers. 2017 Jun;3(1):17038.

2 Rosier BT, Marsh PD, Mira A. Resilience of the oral microbiota in health: mechanisms that prevent dysbiosis. J Dent Res. 2018 Apr; 97(4):371-80.

3 Graves DT, Corrêa JD, Silva TA. The oral microbiota is modified by systemic diseases. J Dent Res. 2019 Feb;98(2):148-56.

4 Jia G, Zhi A, Lai PF, Wang G, Xia Y, Xiong Z, et al. The oral microbiota - a mechanistic role for systemic diseases. Br Dent J. 2018 Mar; 224(6):447-55

5 Nikitakis NG, Papaioannou W, Sakkas LI, Kousvelari E. The autoimmunity-oral microbiome connection. Oral Dis. 2017 Oct;23(7): 828-39.

6 van der Meulen TA, Harmsen H, Bootsma H, Spijkervet F, Kroese F, Vissink A. The microbiome-systemic diseases connection. Oral Dis. 2016 Nov;22(8):719-34.

7 Fåk F, Tremaroli V, Bergström G, Bäckhed F. Oral microbiota in patients with atherosclerosis. Atherosclerosis. 2015 Dec;243(2):5738 .

8 Koren O, Spor A, Felin J, Fåk F, Stombaugh J, Tremaroli V, et al. Human oral, gut, and plaque microbiota in patients with atherosclerosis. Proc Natl Acad Sci USA. 2011 Mar; 108(Suppl 1):4592-8.

9 Hyvärinen K, Mäntylä P, Buhlin K, Paju S, Nieminen MS, Sinisalo J, et al. A common periodontal pathogen has an adverse association with both acute and stable coronary artery disease. Atherosclerosis. 2012 Aug;223(2): 478-84.

10 Pietiäinen M, Liljestrand JM, Kopra E, Pussinen PJ. Mediators between oral dysbiosis and cardiovascular diseases. Eur J Oral Sci. 2018 Oct;126(Suppl 1):26-36

11 Long J, Cai Q, Steinwandel M, Hargreaves MK, Bordenstein SR, Blot WJ, et al. Association of oral microbiome with type 2 diabetes risk. J Periodontal Res. 2017 Jun;52(3):63643.

12 Tam J, Hoffmann T, Fischer S, Bornstein S, Gräßler J, Noack B. Obesity alters composition and diversity of the oral microbiota in patients with type 2 diabetes mellitus independently of glycemic control. PLoS One. 2018 Oct;13(10):e0204724.

13 Scher JU, Ubeda C, Equinda M, Khanin R, Buischi Y, Viale A, et al. Periodontal disease and the oral microbiota in new-onset rheumatoid arthritis. Arthritis Rheum. 2012 Oct 64(10):3083-94.

14 Zhang X, Zhang D, Jia H, Feng Q, Wang D, Liang $\mathrm{D}$, et al. The oral and gut microbiomes are perturbed in rheumatoid arthritis and partly normalized after treatment. Nat Med. 2015 Aug;21(8):895-905.
15 Corrêa JD, Calderaro DC, Ferreira GA, Mendonça SM, Fernandes GR, Xiao E, et al. Subgingival microbiota dysbiosis in systemic lupus erythematosus: association with periodontal status. Microbiome. 2017 Mar;5(1): 34.

16 van der Meulen TA, Harmsen HJ, Vila AV, Kurilshikov A, Liefers SC, Zhernakova A, et al. Shared gut, but distinct oral microbiota composition in primary Sjögren's syndrome and systemic lupus erythematosus. J Autoimmun. 2019 Feb;97:77-87.

17 de Paiva CS, Jones DB, Stern ME, Bian F, Moore QL, Corbiere S, et al. Altered mucosal microbiome diversity and disease severity in Sjögren syndrome. Sci Rep. 2016 Apr;6(1): 23561.

18 van der Meulen TA, Harmsen HJ, Bootsma H, Liefers SC, Vich Vila A, Zhernakova A, et al. Dysbiosis of the buccal mucosa microbiome in primary Sjögren's syndrome patients. Rheumatology (Oxford). 2018 Dec;57(12): 2225-34.

19 Akiyama T, Seishima M, Watanabe H, Nakatani A, Mori S, Kitajima Y. The relationships of onset and exacerbation of pustulosis palmaris et plantaris to smoking and focal infections. J Dermatol. 1995 Dec;22(12):930-4

20 Eriksson MO, Hagforsen E, Lundin IP, Michaëlsson G. Palmoplantar pustulosis: a clinical and immunohistological study. Br J Dermatol. 1998 Mar;138(3):390-8.

21 Hosoki M, Bando E, Asaoka K, Takeuchi H, Nishigawa K. Assessment of allergic hypersensitivity to dental materials. Biomed Mater Eng. 2009;19(1):53-61.

22 Nakamura K, Imakado S, Takizawa M, Adachi M, Sugaya M, Wakugawa M, et al. Exacerbation of pustulosis palmaris et plantaris after topical application of metals accompanied by elevated levels of leukotriene B4 in pustules. J Am Acad Dermatol. 2000 Jun;42(6):1021-5.

23 Kikuchi N, Yamamoto T. Dental infection as a triggering factor in palmoplantar pustulosis. Acta Derm Venereol. 2013 Nov;93(6):721-2.

24 Kouno M, Nishiyama A, Minabe M, Iguchi N, Ukichi K, Nomura T, et al. Retrospective analysis of the clinical response of palmoplantar pustulosis after dental infection control and dental metal removal. J Dermatol. 2017 Jun; 44(6):695-8.

25 Murai O, Sasaki D, Ando Y, Fujimura A, Oikawa $\mathrm{H}$, Suwa $\mathrm{N}$, et al. Improvement of pustulosis palmaris et plantaris by periodontal infection control in a patient with chronic periodontitis. Clin Lab. 2012;58(3-4):323-7.

26 Camelo-Castillo AJ, Mira A, Pico A, Nibali L, Henderson B, Donos N, et al. Subgingival microbiota in health compared to periodontitis and the influence of smoking. Front Microbiol. 2015 Feb;6:119.
27 Colombo AP, Bennet S, Cotton SL, Goodson JM, Kent R, Haffajee AD, et al. Impact of periodontal therapy on the subgingival microbiota of severe periodontitis: comparison between good responders and individuals with refractory periodontitis using the human oral microbe identification microarray. J Periodontol. 2012 Oct;83(10):1279-87.

28 Dingsdag S, Nelson S, Coleman NV. Bacterial communities associated with apical periodontitis and dental implant failure. Microb Ecol Health Dis. 2016 Nov;27:31307.

29 Schaumann S, Staufenbiel I, Scherer R, Schilhabel M, Winkel A, Stumpp SN, et al. Pyrosequencing of supra- and subgingival biofilms from inflamed peri-implant and periodontal sites. BMC Oral Health. 2014 Dec;14(1):157.

30 Camelo-Castillo A, Novoa L, Balsa-Castro C, Blanco J, Mira A, Tomás I. Relationship between periodontitis-associated subgingival microbiota and clinical inflammation by $16 \mathrm{~S}$ pyrosequencing. J Clin Periodontol. 2015 Dec;42(12):1074-82.

31 Donati C, Zolfo M, Albanese D, Tin Truong D, Asnicar F, Iebba V, et al. Uncovering oral Neisseria tropism and persistence using metagenomic sequencing. Nat Microbiol. 2016 May; 1(7):16070.

32 Meuric V, Le Gall-David S, Boyer E, AcuñaAmador L, Martin B, Fong SB, et al. Signature of microbial dysbiosis in periodontitis. Appl Environ Microbiol. 2017 Jun;83(14):e0046217.

33 Takeshita T, Nakano Y, Kumagai T, Yasui M, Kamio N, Shibata Y, et al. The ecological proportion of indigenous bacterial populations in saliva is correlated with oral health status. ISME J. 2009 Jan;3(1):65-78.

34 Wang Y, Zhang J, Chen X, Jiang W, Wang S, $\mathrm{Xu} \mathrm{L}$, et al. Profiling of oral microbiota in early childhood caries using single-molecule real-time sequencing. Front Microbiol. 2017 Nov; $8: 2244$.

35 Muto M, Hitomi Y, Ohtsu A, Shimada H, Kashiwase Y, Sasaki H, et al. Acetaldehyde production by non-pathogenic Neisseria in human oral microflora: implications for carcinogenesis in upper aerodigestive tract. Int J Cancer. 2000 Nov;88(3):342-50.

36 Yokoyama S, Takeuchi K, Shibata Y, Kageyama S, Matsumi R, Takeshita T, et al. Characterization of oral microbiota and acetaldehyde production. J Oral Microbiol. 2018 Jul; 10(1):1492316.

37 Koopman JE, Buijs MJ, Brandt BW, Keijser BJ, Crielaard W, Zaura E. Nitrate and the origin of saliva influence composition and short chain fatty acid production of oral microcosms. Microb Ecol. 2016 Aug;72(2):479-92.

38 Vanhatalo A, Blackwell JR, L'Heureux JE, Williams DW, Smith A, van der Giezen M, et al. Nitrate-responsive oral microbiome modulates nitric oxide homeostasis and blood pressure in humans. Free Radic Biol Med. 2018 Aug; 124:21-30. 
39 Koyanagi T, Sakamoto M, Takeuchi Y, Ohkuma M, Izumi Y. Analysis of microbiota associated with peri-implantitis using $16 \mathrm{~S}$ rRNA gene clone library. J Oral Microbiol. 2010 May;2.

40 You M, Mo S, Watt RM, Leung WK. Prevalence and diversity of Synergistetes taxa in periodontal health and disease. J Periodontal Res. 2013 Apr;48(2):159-68.

41 Yu XL, Chan Y, Zhuang LF, Lai HC, Lang NP, Lacap-Bugler DC, et al. Distributions of Synergistetes in clinically-healthy and diseased periodontal and peri-implant niches. Microb Pathog. 2016 May;94:90-103.

42 Belibasakis GN, Oztürk VO, Emingil G, Bostanci N. Synergistetes cluster A in saliva is associated with periodontitis. J Periodontal Res. 2013 Dec;48(6):727-32.
43 Hugenholtz P, Hooper SD, Kyrpides NC. Focus: synergistetes. Environ Microbiol. 2009 Jun;11(6):1327-9.

44 Vartoukian SR, Palmer RM, Wade WG. Cultivation of a Synergistetes strain representing a previously uncultivated lineage. Environ Microbiol. 2010 Apr;12(4):916-28.

45 Marchesan JT, Morelli T, Moss K, Barros SP, Ward M, Jenkins W, et al. Association of Synergistetes and cyclodipeptides with periodontitis. J Dent Res. 2015 Oct;94(10):1425-31.

46 Mayanagi G, Kimura M, Nakaya S, Hirata H, Sakamoto M, Benno Y, et al. Probiotic effects of orally administered Lactobacillus salivarius WB21-containing tablets on periodontopathic bacteria: a double-blinded, placebo-controlled, randomized clinical trial. J Clin Periodontol. 2009 Jun;36(6):506-13.
47 Iniesta $\mathrm{M}$, Herrera D, Montero E, Zurbriggen M, Matos AR, Marín MJ, et al. Probiotic effects of orally administered Lactobacillus reuteri-containing tablets on the subgingival and salivary microbiota in patients with gingivitis. A randomized clinical trial. J Clin Periodontol. 2012 Aug;39(8):736-44.

48 Alanzi A, Honkala S, Honkala E, Varghese A, Tolvanen M, Söderling E. Effect of Lactobacillus rhamnosus and Bifidobacterium lactis on gingival health, dental plaque, and periodontopathogens in adolescents: a randomised placebo-controlled clinical trial. Benef Microbes. 2018 Jun;9(4):593-602.

49 Olsen I, Yamazaki K. Can oral bacteria affect the microbiome of the gut? J Oral Microbiol. 2019 Mar;11(1):1586422. 